\title{
Computation of the Para-Pseudo Inverse for Oversampled Filter Banks: Forward and Backward Greville Formulas
}

\author{
Lu Gan, Member, IEEE, Cong Ling, Member, IEEE
}

\begin{abstract}
Frames and oversampled filter banks have been extensively studied over the past few years due to their increased design freedom and improved error resilience. In frame expansions, the least square signal reconstruction operator is called the dual frame, which can be obtained by choosing the synthesis filter bank as the para-pseudo inverse of the analysis bank. In this paper, we study the computation of the dual frame by exploiting the Greville formula, which was originally derived in 1960 to compute the pseudo inverse of a matrix when a new row is appended. Here, we first develop the backward Greville formula to handle the case of row deletion. Based on the forward Greville formula, we then study the computation of para-pseudo inverse for extended filter banks and Laplacian pyramids. Through the backward Greville formula, we investigate the frame-based error resilient transmission over erasure channels. The necessary and sufficient condition for an oversampled filter bank to be robust to one erasure channel is derived. A post-filtering structure is also presented to implement the para-pseudo inverse when the transform coefficients in one subband are completely lost.
\end{abstract}

Index Terms - Frame expansions, para-pseudo inverse, dual frame, oversampled filter banks, Laplacian pyramid, Greville formulas.

\section{INTRODUCTION}

In many engineering applications, it is well known that redundancy can offer improved resilience and stability. The concept of redundant signal representations through frame expansions was first introduced by Duffin and Schaeffer in [1]. The wavelet frames have been popularized since 1980s due to the work by Daubechies, Grossman and Meyer [2]. Later in [3] and [4], it was shown that frames in $l^{2}(\mathbb{Z})$ can be implemented via perfect reconstruction (PR) oversampled (O) filter banks (FBs). The discovery of such connections triggered an extensive study of various OFBs, such as the DFT-based systems [5], [6], cosine-modulated systems [7], [8] and linear-phase systems [9]-[11]. In the mean time, the structural redundancy in frame expansions has also been exploited for various applications such as quantization, errorresilient multimedia coding [12]-[14], segmentation, pattern recognition, wireless communications, and sensor networks. For a more complete overview of frame theory and OFBs, see the introductory paper [15], [16] and the references therein.

This work was published in part in the Proceedings of IEEE International Conference on Acoustics, Speech and Signal Processing, Honolulu, Hawaii, US, April 2007.

L. Gan is with the School of Engineering and Design, Brunel University, London UB8 3PH, United Kingdom (email: lu.gan@brunel.ac.uk).

C. Ling is with the Department of Electrical and Electronic Engineering, Imperial College London, London SW7 2AZ, United Kingdom (email: cling@ieee.org).
Unlike conventional non-redundant bases, there are many operators that can reconstruct a signal from its frame coefficients. Among them, the most significant one is the dual frame $^{1}$, which offers the optimal solution in the least square sense. The dual frame of a finite-dimensional frame in $\mathbb{C}^{M}$ corresponds to its pseudo inverse. In $l^{2}(\mathbb{Z})$, the dual frame can be implemented via the para-pseudo inverse of the analysis bank. While the dual frame of a tight frame is just itself, for a general frame in $l^{2}(\mathbb{Z})$, the explicit computation of the dual frame is a challenging task as it requires the inversion of a polynomial matrix. If the frame has a shift-invariant structure [4], we can approximate the dual frame using truncated Neumann series expansion [2], [4]. However, such an approximation could be computationally inefficient and numerically unstable. In [17], Li et al. proposed a state-space approach to calculate the dual frame. Although this method is numerically stable, it does not offer a fast implementation structure or closed-form solution.

Recall that a frame can be constructed either by adding additional rows to a basis [18], or by deleting some columns of a basis in a larger space [15], [16]. Thus, it is natural to ponder whether the dual frame can be calculated from the dual basis. Bearing this in mind, we aim to exploit the Greville formula, which was originally proposed in [19] to obtain the pseudo inverse of a matrix when it is augmented by a row (or column) vector. Due to its efficiency, the Greville formula has been used as a benchmark for the calculation of pseudo inverse. It has also found wide applications in database and neural network computation [20]. In this paper, the backward Greville formula is further developed to compute the pseudo inverse when a row (or column) of a matrix is deleted. This is also motivated by the application of frames in error-resilient transmission [12], [21], where efficient updating of the dual frame is needed when some communication channels are completely down. Then, based on the forward and backward Greville formulas, we study the following frames and OFBs:

- Extended filter banks: This subclass of OFBs are obtained by adding one or more filters to an existing PR FB [18]. They are quite useful in unequal error protection and joint source-channel coding [18]. By exploiting the forward Greville formula, we propose a recursive method to calculate their dual frame. We also derive a necessary and sufficient condition to generate doubly finite impulse response (DFIR) systems [18], where all the analysis and

\footnotetext{
${ }^{1}$ Throughout this paper, the term dual frame is referred to as the reconstruction operator with minimum $l_{2}$ norm.
} 
dual synthesis filters are FIR. Not only does this condition offer efficient design of DFIR extended FBs, it also leads to hardware friendly implementation structures.

- Laplacian pyramids (LPs): The LP was first proposed in [22] as a signal processing tool for multi-resolution representation of images. Recently, Do et al. [23] conducted a frame-theoretical analysis of such systems. Here, we derive the closed-form solution of the LP's dual frame based on the forward Greville formula. The necessary and sufficient condition to generate a DFIR LP frame is also given.

- OFBs for erasure channels: We show that the backward Greville formula facilitates the analysis of erasure resilience of OFBs. In particular, we establish the necessary and sufficient condition for an OFB robust to one erasure channel. We also propose a post-filtering structure to implement the para-pseudo inverse of the remaining analysis bank when the transform coefficients in one subband are completely lost.

The rest of this paper is organized as follows. In Section II, we introduce the notations and review the basics of frame theory and OFBs. In Section III, we present the forward Greville formula and derive the new backward Greville formula. Applications of the forward Greville formula are then demonstrated in Section IV and Section V, where we investigate the extended FBs and LP frames, respectively. Section VI is dedicated to the analysis of OFBs for erasure channels, followed by conclusions in Section VII.

\section{PRELIMINARIES}

\section{A. Notations}

Bold-faced letters indicate vectors and matrices. The symbol $\mathbf{I}_{M}$ denotes an $M \times M$ identity matrix. The size of a matrix is omitted when it is clear from the context. The superscript $H$ represents the Hermitian transpose. For a rational polynomial $e(z), \tilde{e}(z)$ denotes the function which conjugates the coefficients, and then replaces $z$ with $z^{-1}$. Similarly, for a rational polynomial matrix $\mathbf{E}(z), \tilde{\mathbf{E}}(z)$ represents the matrix obtained from $\mathbf{E}(z)$ by transposing it, conjugating all coefficients of the rational functions in $\mathbf{E}(z)$, and replacing $z$ by $z^{-1}$. We say that an $1 \times M$ row vector $\mathbf{e}(z)$ is paraunitary $(\mathrm{PU})$ if $\mathbf{e}(z) \tilde{\mathbf{e}}(z)=A$ for a positive constant $A$. Likewise, an $N \times M(N \geq M)$ matrix $\mathbf{E}(z)$ is said to be PU if it satisfies $\tilde{\mathbf{E}}(z) \mathbf{E}(z)=A \mathbf{I}_{M}$. The para-pseudo inverse of $\mathbf{E}(z)$ is denoted by $\mathbf{E}^{\dagger}(z)$. Just as the conventional pseudo inverse, the para-pseudo inverse is the unique solution of the following Moore-Penrose equations [24]:

$$
\begin{gathered}
\mathbf{E}(z) \mathbf{E}^{\dagger}(z) \mathbf{E}(z)=\mathbf{E}(z) \\
\mathbf{E}^{\dagger}(z) \mathbf{E}(z) \mathbf{E}^{\dagger}(z)=\mathbf{E}^{\dagger}(z) \\
\tilde{\mathbf{E}}^{\dagger}(z) \tilde{\mathbf{E}}(z)=\mathbf{E}(z) \mathbf{E}^{\dagger}(z) \\
\tilde{\mathbf{E}}(z) \tilde{\mathbf{E}}^{\dagger}(z)=\mathbf{E}^{\dagger}(z) \mathbf{E}(z)
\end{gathered}
$$

In (3) and (4), $\tilde{\mathbf{E}}^{\dagger}(z)$ is defined as $\tilde{\mathbf{E}}^{\dagger}(z)=[\tilde{\mathbf{E}}(z)]^{\dagger}$. It is worth mentioning that the operations of " $\nmid$ " and " $"$ are permutable. Also, note that when $\mathbf{E}(z)$ is a constant zero-order matrix or when $z=e^{j \omega}, \mathbf{E}^{\dagger}(z)$ reduces to the conventional pseudo inverse.

\section{B. Frame Theory and $O F B$ s}

Consider a set of vectors $\Phi=\left\{\varphi_{i}\right\}_{i \in \mathbb{Z}}$ in a Hilbert space $\mathbb{H}$. $\Phi$ is called a frame if for any signal $x$, there exist two positive constants $0<A \leq B<\infty$ so that

$$
A\|x\|^{2} \leq \sum_{i \in \mathbb{Z}}\left|y_{i}\right|^{2} \leq B\|x\|^{2}
$$

where the $i$-th frame coefficient $y_{i}=\left\langle x, \varphi_{i}\right\rangle$ is the inner product of $x$ and $\varphi_{i}$. In (5), the constants $A$ and $B$ are called frame bounds. When $A=B$, we say that $\Phi$ generates a tight frame and it is Parseval-tight if $A=B=1$. Moreover, $\Phi$ is a uniform tight frame (UTF) if $\left\|\varphi_{i}\right\|^{2}=1$ for $i \in \mathbb{Z}$ [15], [16].

Given a frame $\Phi$, there exists a reconstruction frame $\Psi=$ $\left\{\psi_{i}\right\}_{i=1}^{N}$ that can recover the signal $x$ from its frame coefficients:

$$
x=\sum_{i \in \mathbb{Z}} y_{i} \psi_{i}
$$

Note that for a given frame $\Phi$, there are many solutions of $\Psi$. A particular one is the dual frame, which corresponds to the least square solution. If $\Phi$ is a tight frame, it can be shown that $\Psi=\frac{1}{A} \Phi[15],[16]$.

From a signal-processing point of view, a frame in $l^{2}(\mathbb{Z})$ corresponds to an $N$-channel PR OFB with sampling factor $M(N \geq M)$, as shown in Fig. 1(a). Here, $H_{i}(z)=$ $\sum_{n=-\infty}^{\infty} h_{i}[n] z^{-n}$ and $F_{i}(z)=\sum_{n=-\infty}^{\infty} f_{i}[n] z^{-n}$ (for $i=$ $1, \cdots, N)$ represent the $i$-th analysis and synthesis filters, respectively. Define $h_{i, j}[k]=h_{i}[j M-k]$. The signal decomposition in an OFB can be described as

$$
y_{i}[j]=\sum_{k=-\infty}^{\infty} x[k] h_{i}[j M-k]=\left\langle x, h_{i, j}\right\rangle .
$$

Accordingly, the reconstruction formula takes the form of

$$
\hat{x}[n]=\sum_{i=1}^{N} \sum_{k=-\infty}^{\infty} y_{i}[k] f_{i}[n-M k] .
$$

The polyphase representation of an OFB is shown in Fig. 1(b), where the $N \times M$ polynomial matrix $\mathbf{E}(z)$ and the $M \times N$ polynomial matrix $\mathbf{R}(z)$ denote the analysis and synthesis polyphase matrices, respectively. The system is called "oversampled" as the number of channels $N$ is greater than the sampling factor $M$. Hence, $\mathbf{E}(z)$ is a tall matrix, while $\mathbf{R}(z)$ is a fat one. The OFB implements a frame expansion in $l^{2}(\mathbb{Z})$ if it has the PR property [25]

$$
\mathbf{R}(z) \mathbf{E}(z)=\mathbf{I}_{M} .
$$

Besides, $\mathbf{E}(z)$ corresponds to a tight frame with frame bound $A$ if and only if it is a PU matrix satisfying $\tilde{\mathbf{E}}(z) \mathbf{E}(z)=A \mathbf{I}_{M}$. Note that (9) implies that $\mathbf{R}(z)$ should be a left inverse of $\mathbf{E}(z)$. Among all left inverses, the one that corresponds to the dual frame is the para-pseudo inverse [4]

$$
\mathbf{E}^{\dagger}(z)=(\tilde{\mathbf{E}}(z) \mathbf{E}(z))^{-1} \tilde{\mathbf{E}}(z) .
$$




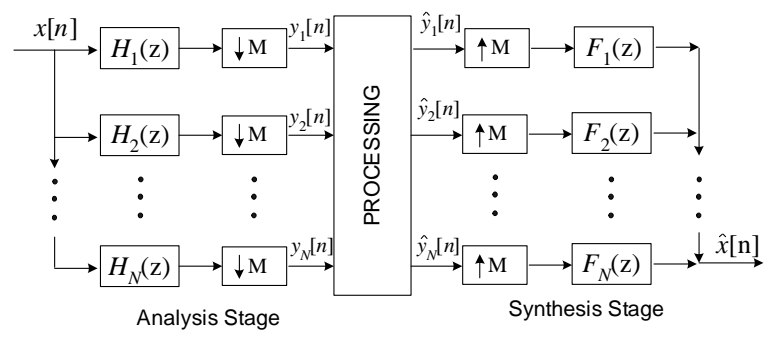

(a)

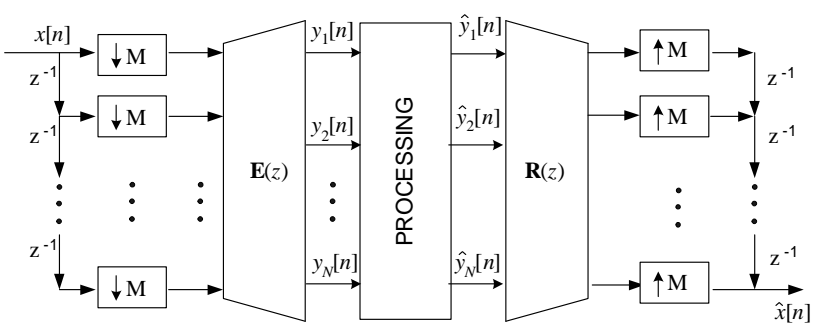

(b)

Fig. 1. An $N$-channel oversampled filter bank with sampling factor of $M$. (a) Direct-form implementation; (b) Polyphase representation.

Obviously, for a PU OFB satisfying $\tilde{\mathbf{E}}(z) \mathbf{E}(z)=A \mathbf{I}_{M}$, $\mathbf{E}^{\dagger}(z)=\frac{1}{A} \tilde{\mathbf{E}}(z)$. However, for a general PR system, direct computation of $\mathbf{E}^{\dagger}(z)$ involves the costly inversion of the rational polynomial matrix $\tilde{\mathbf{E}}(z) \mathbf{E}(z)$. In what follows, we aim to address the computation of $\mathbf{E}^{\dagger}(z)$ using the forward and backward Greville formulas presented in the next section.

\section{FORWARD AND BACKWARD GREVILLE FORMULAS}

\section{A. Forward Greville Formula}

The original Greville formula in [19] was developed for zero-order matrices. Here, it is adapted to accommodate rational polynomial matrices. Suppose that $\mathbf{E}_{i}(z)$ is an $i \times$ $M$ analysis polyphase matrix consisting of $i$ analysis filters $H_{1}(z), H_{2}(z), \cdots, H_{i}(z)$. Partition it into

$$
\mathbf{E}_{i}(z)=\left[\begin{array}{c}
\mathbf{E}_{i-1}(z) \\
\mathbf{e}_{i}(z)
\end{array}\right]
$$

where $\mathbf{E}_{i-1}(z)$ is the $(i-1) \times M$ submatrix and $\mathbf{e}_{i}(z)$ is its last row. Assume further that we know $\mathbf{E}_{i-1}^{\dagger}(z)$. The Greville formula [19] computes $\mathbf{E}_{i}^{\dagger}(z)$ from $\mathbf{E}_{i-1}^{\dagger}(z)$ and $\mathbf{e}_{i}(z)$ as follows:

Set

$$
\mathbf{d}_{i}(z)=\mathbf{e}_{i}(z) \mathbf{E}_{i-1}^{\dagger}(z)
$$

and

$$
\mathbf{c}_{i}(z)=\mathbf{e}_{i}(z)-\mathbf{d}_{i}(z) \mathbf{E}_{i-1}(z) ;
$$

If $\mathbf{c}_{i}(z)=\mathbf{0}$ for all $z$ (Case 1 ), set

$$
\mathbf{r}_{i}(z)=\frac{\mathbf{d}_{i}(z)}{1+\mathbf{d}_{i}(z) \tilde{\mathbf{d}}_{i}(z)} \tilde{\mathbf{E}}_{i-1}^{\dagger}(z) ;
$$

otherwise (Case 2), set

$$
\mathbf{r}_{i}(z)=\frac{\mathbf{c}_{i}(z)}{\mathbf{c}_{i}(z) \tilde{\mathbf{c}}_{i}(z)},
$$

then

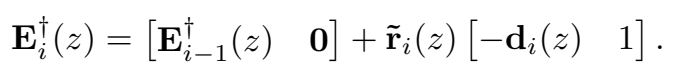

Remarks:

1) While we only present here the formula for row addition, column addition can be easily handled. Details can be found in [19] and [26].

2) For any row vector $\mathbf{e}(z)$, its para-pseudo inverse $\mathbf{e}^{\dagger}(z)$ can be expressed as

$$
\mathbf{e}^{\dagger}(z)=\frac{\tilde{\mathbf{e}}(z)}{\mathbf{e}(z) \tilde{\mathbf{e}}(z)} .
$$

Thus, to compute $\mathbf{E}_{i}^{\dagger}(z)$, one can first calculate the para-pseudo inverse of its first row vector $\mathbf{e}_{1}(z)$ and then iteratively apply the forward Greville formula for $i-1$ times. In each iteration, both $\mathbf{d}_{i}(z)$ and $\mathbf{c}_{i}(z)$ are rational polynomial row vectors. Hence, it avoids the costly polynomial matrix inversion required in (10).

3) In Case $1, \mathbf{c}_{i}(z)$ is a constant zero vector, which implies that there exists a rational polynomial row vector $\mathbf{d}_{i}(z)$ so that

$$
\mathbf{e}_{i}(z)=\mathbf{d}_{i}(z) \mathbf{E}_{i-1}(z) .
$$

While in case 2 , such a rational polynomial row vector does not exist. In the special case when $\mathbf{E}_{i}(z)$ is a zeroorder matrix, i.e., when $\mathbf{E}_{i}(z)=\mathbf{E}_{i}$, (18) reduces to $\mathbf{e}_{i}=\mathbf{d}_{i} \mathbf{E}_{i-1}$, which indicates that $\mathbf{e}_{i}$ lies in the row space of $\mathbf{E}_{i-1}$ for Case 1, and not for Case 2 [26].

Example 1 (Forward Greville Formula): This example demonstrates the computation of the para-pseudo inverse through the forward Greville formula. Consider a $3 \times 2$ polynomial matrix as follows

$$
\mathbf{E}_{3}(z)=\left[\begin{array}{cc}
1 & 0 \\
1 & z-a \\
0 & 1
\end{array}\right]
$$

where $a$ is a real number.

We first calculate the para-pseudo inverse of

$$
\mathbf{E}_{2}(z)=\left[\begin{array}{l}
\mathbf{e}_{1}(z) \\
\mathbf{e}_{2}(z)
\end{array}\right]=\left[\begin{array}{cc}
1 & 0 \\
1 & z-a
\end{array}\right]
$$

By (17), we know that $\mathbf{e}_{1}^{\dagger}(z)=\left[\begin{array}{ll}1 & 0\end{array}\right]^{T}$. Hence, from (12) and (13), we get $\mathbf{d}_{2}(z)=\mathbf{e}_{2}(z) \mathbf{e}_{1}^{\dagger}(z)=1$ and $\mathbf{c}_{2}(z)=\mathbf{e}_{2}(z)-$ $\mathbf{d}_{2}(z) \mathbf{E}_{2}(z)=\left[\begin{array}{ll}0 & z-a\end{array}\right]$. As $\mathbf{c}_{2}(z)$ is not a constant zero vector (Case 2), we apply (15) to get

$$
\mathbf{r}_{2}(z)=\frac{1}{(z-a)\left(z^{-1}-a\right)}\left[\begin{array}{ll}
0 & z-a
\end{array}\right]
$$

Substituting $\mathbf{e}_{1}^{\dagger}(z), \mathbf{r}_{2}(z)$ and $\mathbf{d}_{2}(z)$ into (16) leads to

$$
\mathbf{E}_{2}^{\dagger}(z)=\left[\begin{array}{cc}
1 & 0 \\
-\frac{1}{z-a} & \frac{1}{z-a}
\end{array}\right]
$$

We then move on to calculate $\mathbf{E}_{3}^{\dagger}(z)$. Again, by definition of $(12), \mathbf{d}_{3}(z)=\mathbf{e}_{3}(z) \mathbf{E}_{2}^{\dagger}(z)=\frac{1}{z-a}\left[\begin{array}{ll}-1 & 1\end{array}\right]$. Note that as $\mathbf{E}_{2}^{\dagger}(z) \mathbf{E}_{2}(z)=\mathbf{I}$, we have $\mathbf{c}_{3}(z)=\left[\begin{array}{ll}0 & 0\end{array}\right]$ (Case 1). Substituting $\mathbf{d}_{3}(z)$ into (14) produces $\mathbf{r}_{3}(z)=\frac{1}{\gamma(z)}\left[\begin{array}{ll}a-z & 2\end{array}\right]$, 
where $\gamma(z)=2+(z-a)\left(z^{-1}-a\right)=3-a z^{-1}-a z+a^{2}$. Finally, by (16), we arrive at the following expression

$$
\mathbf{E}_{3}^{\dagger}(z)=\frac{1}{\gamma(z)}\left[\begin{array}{ccc}
\gamma(z)-1 & 1 & a-z \\
a-z^{-1} & z^{-1}-a & 2
\end{array}\right] .
$$

One can check that $\mathbf{E}_{3}^{\dagger}(z)$ given above satisfies the MoorePenrose equations (1)-(4).

\section{B. Backward Greville Formula}

Now, let us consider the reverse problem. Suppose we know $\mathbf{E}_{i}(z)$ and $\mathbf{E}_{i}^{\dagger}(z)$, how to calculate $\mathbf{E}_{i-1}^{\dagger}(z)$ ? As can be seen from the forward Greville formula, we first need to distinguish between two cases. To do this, we see from (16) that $\tilde{\mathbf{r}}_{i}(z)$ is the last column of $\mathbf{E}_{i}^{\dagger}(z)$, and so we can similarly partition $\mathbf{E}_{i}^{\dagger}(z)$ in the form

$$
\mathbf{E}_{i}^{\dagger}(z)=\left[\begin{array}{ll}
\mathbf{R}_{i-1}(z) & \tilde{\mathbf{r}}_{i}(z)
\end{array}\right]
$$

where $\mathbf{R}_{i-1}(z)$ is the $M \times(i-1)$ submatrix and $\tilde{\mathbf{r}}_{i}(z)$ is the $M \times 1$ column vector. According to [19] and [26],

$$
\mathbf{e}_{i}(z) \tilde{\mathbf{r}}_{i}(z)= \begin{cases}\frac{\mathbf{d}_{i}(z) \tilde{\mathbf{d}}_{i}(z)}{1+\mathbf{d}_{i}(z) \tilde{\mathbf{d}}_{i}(z)}, & \text { Case 1; } \\ 1, & \text { Case 2. }\end{cases}
$$

Thus, it is clear that the two cases can easily be distinguished by checking whether $\mathbf{e}_{i}(z) \tilde{\mathbf{r}}_{i}(z)$ is a constant unit. The backward Greville algorithm is presented in Theorem 1 below.

Theorem 1 (Backward Greville Formula): Let $\mathbf{E}_{i}(z)$ and $\mathbf{E}_{i}^{\dagger}(z)$ be partitioned as in (11) and (20), respectively. If $\mathbf{e}_{i}(z) \tilde{\mathbf{r}}_{i}(z)$ is not a constant 1 (Case 1), set

$$
\mathbf{d}_{i}(z)=\frac{\mathbf{e}_{i}(z) \mathbf{R}_{i-1}(z)}{1-\mathbf{e}_{i}(z) \tilde{\mathbf{r}}_{i}(z)}
$$

otherwise, if $\mathbf{e}_{i}(z) \tilde{\mathbf{r}}_{i}(z)=1$ for all $z$ (Case 2), set

$$
\mathbf{d}_{i}(z)=-\frac{\mathbf{r}_{i}(z) \mathbf{R}_{i-1}(z)}{\mathbf{r}_{i}(z) \tilde{\mathbf{r}}_{i}(z)}
$$

The dual frame of $\mathbf{E}_{i-1}(z)$ is given by

$$
\mathbf{E}_{i-1}^{\dagger}(z)=\mathbf{R}_{i-1}(z)+\tilde{\mathbf{r}}_{i}(z) \mathbf{d}_{i}(z) .
$$

Detailed derivations of this theorem can be found in the Appendix. Just as the forward Greville algorithm, the above results can be easily modified to deal with column deletion.

Remark: The backward Greville formula was also investigated in [27]. But the derivations there were limited to zeroorder matrices whose row vectors are independent (a special case of Case 2). On the other hand, Theorem 1 can be applied to any rational polynomial matrices. Hence, our derivations are more general.

Example 2 (Backward Greville Formula): Let the notations be as in Example 1. To have a quick check of the backward Greville algorithm, we shall calculate $\mathbf{E}_{2}^{\dagger}(z)$ from $\mathbf{E}_{3}(z)$ and $\mathbf{E}_{3}^{\dagger}(z)$. By definition, we know that

$$
\mathbf{e}_{3}(z)=\left[\begin{array}{ll}
0 & 1
\end{array}\right], \quad \tilde{\mathbf{r}}_{3}(z)=\frac{1}{\gamma(z)}\left[\begin{array}{c}
a-z \\
2
\end{array}\right]
$$

and

$$
\mathbf{R}_{2}(z)=\frac{1}{\gamma(z)}\left[\begin{array}{cc}
\gamma(z)-1 & 1 \\
a-z^{-1} & z^{-1}-a
\end{array}\right]
$$

As $\mathbf{e}_{3}(z) \tilde{\mathbf{r}}_{3}(z)=\frac{2}{\gamma(z)} \neq 1$, we need to apply (23). It is easy to check that

$$
\mathbf{e}_{3}(z) \mathbf{R}_{2}(z)=\frac{1}{\gamma(z)}\left[a-z^{-1} \quad z^{-1}-a\right]
$$

which leads to

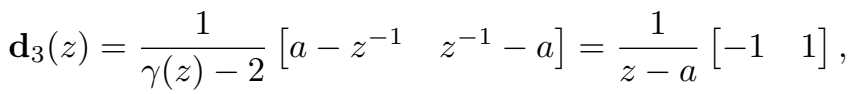

where we have used the equality $\gamma(z)-2=(z-a)\left(z^{-1}-a\right)$. Substituting $\mathbf{d}_{3}(z)$ into (25) produces $\mathbf{E}_{2}^{\dagger}(z)$ as in (19).

In nearly the same manner, by applying Case 2 of the backward Greville formula, one can get $\mathbf{e}_{1}^{\dagger}(z)$ from $\mathbf{E}_{2}(z)$ and $\mathbf{E}_{2}^{\dagger}(z)$. We leave it as an exercise for readers themselves.

\section{Dual Frame of Extended Filter Banks}

\section{A. Computation Formula}

In this Section, we apply the forward Greville formula to study the extended $F B$, which is constructed by adding one or more filters to an existing PR FB. In other words, the $N \times M$ $(N>M)$ polyphase matrix $\mathbf{E}_{N}(z)$ takes the form of

$$
\mathbf{E}_{N}(z)=\left[\begin{array}{c}
\mathbf{E}_{N_{0}}(z) \\
\mathbf{e}_{N_{0}+1}(z) \\
\vdots \\
\mathbf{e}_{N}(z)
\end{array}\right],
$$

where the $N_{0} \times M\left(N_{0} \geq M\right)$ polyphase matrix $\mathbf{E}_{N_{0}}(z)$ has the PR property, and each row vector $\mathbf{e}_{i}(z)\left(N_{0}+1 \leq i \leq\right.$ $N)$ is used to strengthen $\mathbf{E}_{N_{0}}(z)$. Such systems can be used in applications such as unequal error protection and multiple description coding [18]. Recall that extended FBs were first studied in [18]. However, the discussions there were limited to the scenario where $\mathbf{E}_{N_{0}}(z)$ is a critically sampled system (i.e., $N_{0}=M$ ). Here, we allow $N_{0} \geq M$ and hence, $\mathbf{E}_{N_{0}}(z)$ can be either critically sampled or oversampled.

Let us first consider how to calculate $\mathbf{E}_{N}^{\dagger}(z)$ from $\mathbf{E}_{N_{0}}^{\dagger}(z)$. Denote $\mathbf{E}_{i}(z)\left(N_{0}+1 \leq i \leq N\right)$ as the submatrix containing the first $i$ rows of $\mathbf{E}_{N}(z)$ and we partition it as in (11). Since $\mathbf{E}_{N_{0}}(z)$ corresponds to a PR FB, so does $\mathbf{E}_{i-1}(z)\left(N_{0}+1 \leq\right.$ $i \leq N)$, which implies that $\mathbf{E}_{i-1}^{\dagger}(z) \mathbf{E}_{i-1}(z)=\mathbf{I}_{M}$. By (12) and (13), $\mathbf{c}_{i}(z)=\mathbf{0}$. Therefore, we need to apply Case 1 of the forward Greville formula iteratively for $N-M$ times. From (14) and (16), one can easily arrive at the following recursive formula

$$
\mathbf{E}_{i}^{\dagger}(z)=\mathbf{E}_{i-1}^{\dagger}(z) \mathbf{P}_{i}(z), \quad \text { for } i=N_{0}+1 \text { to } N
$$

in which the $(i-1) \times i$ polynomial matrix $\mathbf{P}_{i}(z)$ can be expressed as

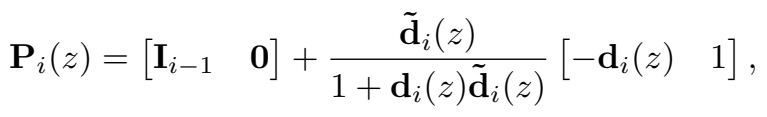

with

$$
\mathbf{d}_{i}(z)=\mathbf{e}_{i}(z) \mathbf{E}_{i-1}^{\dagger}(z) .
$$

Eqs. (27)-(29) imply that $\mathbf{E}_{N}(z)$ can be obtained from $\mathbf{E}_{N_{0}}^{\dagger}(z)$ by post-multiplying it with several matrices $\mathbf{P}_{i}(z)$. Note these 
formulas hold for all FBs, either FIR or IIR, as long as $\mathbf{E}_{N_{0}}(z)$ has the PR property.

Example 3 (Extended oversampled $F B$ ): To demonstrate the application of Eq. (27), let us consider a $3 \times 2$ analysis polyphase matrix $\mathbf{E}_{3}(z)$ as follows

$$
\mathbf{E}_{3}(z)=\left[\begin{array}{cc}
a_{0}(z) & a_{1}(z) \\
a_{0}(z) & -a_{1}(z) \\
b(z) & 0
\end{array}\right]
$$

where $a_{0}(z), a_{1}(z)$ and $b(z)$ are rational polynomials in $z$. The corresponding analysis filters $H_{1}(z), H_{2}(z)$ and $H_{3}(z)$ are low-pass, high-pass and band-pass, respectively. In what follows, we aim to derive the closed-form solution of $\mathbf{E}_{3}^{\dagger}(z)$ using (27). Note that for the first two rows in $\mathbf{E}_{3}(z)$, one can easily calculate its inverse

$$
\mathbf{E}_{2}^{-1}(z)=\frac{1}{2}\left[\begin{array}{cc}
\frac{1}{a_{0}(z)} & \frac{1}{a_{0}(z)} \\
\frac{1}{a_{1}(z)} & -\frac{1}{a_{1}(z)}
\end{array}\right]
$$

As $\mathbf{e}_{3}(z)=\left[\begin{array}{ll}b(z) & 0\end{array}\right]$, we have $\mathbf{d}_{3}(z)=\mathbf{e}_{3}(z) \mathbf{E}_{2}^{-1}(z)=$ $\frac{b(z)}{2 a_{0}(z)}\left[\begin{array}{ll}1 & 1\end{array}\right]$ and $\mathbf{d}_{3}(z) \tilde{\mathbf{d}}_{3}(z)=\frac{b(z) \tilde{b}_{0}(z)}{2 a_{0}(z) \tilde{a}_{0}(z)}$. Substituting them into (27) yields

$\mathbf{E}_{3}^{\dagger}(z)=\frac{1}{2}\left[\begin{array}{ccc}\frac{1}{a_{0}(z)} & \frac{1}{a_{0}(z)} & 0 \\ \frac{1}{a_{1}(z)} & -\frac{1}{a_{1}(z)} & 0\end{array}\right]+\frac{\tilde{b}(z)}{2 a_{0}(z) \tilde{a}_{0}(z)+b(z) \tilde{b}(z)}[-$

It is worthy noting that using the state-space approach, Example 1 of [17] calculated the numerical solution of (30) for a given set of IIR Butterworth filters $H_{i}(z)(1 \leq i \leq 3)$, while the forward Greville formula leads to a closed-form solution.

\section{B. Doubly FIR systems}

One can also see from (28) that $\mathbf{E}_{i}^{\dagger}(z)$ is in general IIR. However, in many applications, the DFIR property is highly desirable where both $\mathbf{E}_{i}(z)$ and $\mathbf{E}_{i}^{\dagger}(z)$ are FIR matrices [18]. Bearing this in mind, we investigate the following problem: Suppose that $\mathbf{E}_{N_{0}}(z)$ in (26) is DFIR, how to design $\mathbf{e}_{i}(z)$ $\left(N_{0}+1 \leq i \leq N\right)$ so that each $\mathbf{E}_{i}(z)$ is also DFIR? It can be readily seen from (27)-(29) that $\mathbf{E}_{i}(z)$ is DFIR if $\mathbf{d}_{i}(z) \tilde{\mathbf{d}}_{i}(z)=d_{i}^{2}$ for some real constant $d_{i}$. But is this condition also necessary? The answer is yes, as presented in the following theorem:

Theorem 2: Define $\mathbf{E}_{i}(z)$ as in (11) and suppose that $\mathbf{E}_{i-1}(z)$ is a DFIR PR FB. Then, $\mathbf{E}_{i}(z)$ is DFIR if and only if $\mathbf{d}_{i}(z)=\mathbf{e}_{i}(z) \mathbf{E}_{i-1}^{\dagger}(z)$ is a PU row vector satisfying

$$
\mathbf{d}_{i}(z) \tilde{\mathbf{d}}_{i}(z)=d_{i}^{2}
$$

for some real constant $d_{i}$. Accordingly, $\mathbf{E}_{i}^{\dagger}(z)$ is given by

$$
\mathbf{E}_{i}^{\dagger}(z)=\mathbf{E}_{i-1}^{\dagger}(z)\left[\mathbf{I}_{M}-\frac{\tilde{\mathbf{d}}_{i}(z) \mathbf{d}_{i}(z)}{1+d_{i}^{2}} \quad \frac{\tilde{\mathbf{d}}_{i}(z)}{1+d_{i}^{2}}\right] .
$$

Proof: Note that (33) can be easily obtained from (27)(29). Hence, we just need to to prove the "if and only if" statement. According to [18], the necessary and sufficient condition for $\mathbf{E}_{i}(z)$ to be a DFIR is $\operatorname{det}\left(\tilde{\mathbf{E}}_{i}(z) \mathbf{E}_{i}(z)\right)=\alpha$ for some non-zero real number $\alpha$. By the matrix determinant lemma, we have

$$
\begin{aligned}
\operatorname{det}\left(\tilde{\mathbf{E}}_{i}(z) \mathbf{E}_{i}(z)\right) & =\operatorname{det}\left(\tilde{\mathbf{E}}_{i-1}(z) \mathbf{E}_{i-1}(z)+\tilde{\mathbf{e}}_{i}(z) \mathbf{e}_{i}(z)\right) \\
& =\left(1+\mathbf{e}_{i}(z) \mathbf{S}_{i-1}^{-1}(z) \tilde{\mathbf{e}}_{i}(z)\right) \cdot \operatorname{det}\left(\mathbf{S}_{i-1}(z)\right),
\end{aligned}
$$

where $\mathbf{S}_{i-1}(z)=\tilde{\mathbf{E}}_{i-1}(z) \mathbf{E}_{i-1}(z)$. Again, since $\mathbf{E}_{i-1}(z)$ is DFIR, det $\left(\mathbf{S}_{i-1}(z)\right)$ is a constant, which implies that $\mathbf{E}_{i}(z)$ is DFIR if and only if $\mathbf{e}_{i}(z) \mathbf{S}_{i-1}^{-1}(z) \tilde{\mathbf{e}}_{i}(z)$ is a constant. When $\mathbf{E}_{i-1}(z)$ has the PR property, it can be verified that

$$
\mathbf{S}_{i-1}^{-1}(z)=\mathbf{E}_{i-1}^{\dagger}(z) \tilde{\mathbf{E}}_{i-1}^{\dagger}(z) .
$$

Hence, by definition of $\mathbf{d}_{i}(z)$, we know that $\mathbf{e}_{i}(z) \mathbf{S}_{i-1}^{-1}(z) \tilde{\mathbf{e}}_{i}(z)=\mathbf{d}_{i}(z) \tilde{\mathbf{d}}_{i}(z)$, which implies that $\mathbf{E}_{i}(z)$ is a DFIR if and only if (32) holds.

The above theorem is a generalized version of Theorem 4 in [18] where $\mathbf{E}_{i-1}(z)$ is restricted to be a critically sampled PU FB only, while in Theorem $2, \mathbf{E}_{i-1}(z)$ can be any DFIR PR FB (including oversampled systems). Moreover, from the design perspective, Theorem 2 offers an efficient way to iterative construction of a DFIR system from (32), as demonstrated in the following example.

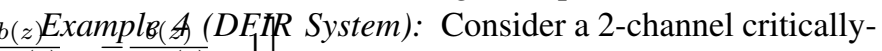
$2 a_{\text {sañ }}$
0

$$
\mathbf{E}_{2}(z)=\left[\begin{array}{l}
\mathbf{e}_{1}(z) \\
\mathbf{e}_{2}(z)
\end{array}\right]
$$

where $\mathbf{e}_{1}(z)$ and $\mathbf{e}_{2}(z)$ correspond to a low-pass and a highpass filter, respectively. Let us first add a new analysis filter $H_{3}(z)$ which is exactly the same as the lowpass one, i.e., $\mathbf{E}_{3}(z)=\left[\begin{array}{l}\mathbf{E}_{2}(z) \\ \mathbf{e}_{1}(z)\end{array}\right]$. By doing so, the resulting OFB can be viewed as an unequal error protection code since the output of the low-pass filter is sent twice. Now, from the Greville formula, $\mathbf{d}_{3}(z)=\mathbf{e}_{1}(z) \mathbf{E}_{2}(z)=\left[\begin{array}{ll}1 & 0\end{array}\right]$. Hence, $\mathbf{d}_{3}(z) \tilde{\mathbf{d}}_{3}(z)=1$ and by (27), we have

$$
\mathbf{E}_{3}^{\dagger}(z)=\mathbf{E}_{2}^{-1}(z)\left[\begin{array}{ccc}
\frac{1}{2} & 0 & \frac{1}{2} \\
0 & 1 & 0
\end{array}\right] .
$$

Next, we try to add another filter $\mathbf{e}_{4}(z)$ so that the $4 \times 2$ polyphase matrix $\mathbf{E}_{4}(z)=\left[\begin{array}{l}\mathbf{E}_{3}(z) \\ \mathbf{e}_{4}(z)\end{array}\right]$ is also DFIR. From Theorem 2, $\mathbf{E}_{4}(z)$ can be DFIR if $\mathbf{d}_{4}(z)$ is a PU row vector. At the first glance, it seems that $\mathbf{e}_{4}(z)$ could be directly obtained from (18) by choosing $\mathbf{d}_{4}(z)$ as a PU row vector. However, it should be emphasized that as $\mathbf{E}_{3}(z)$ is a tall matrix, $\mathbf{E}_{3}(z) \mathbf{E}_{3}^{\dagger}(z) \neq \mathbf{I}_{3}$. Hence, $\mathbf{d}_{4}(z) \mathbf{E}_{3}(z) \mathbf{E}_{3}^{\dagger}(z)$ may not be a PU row vector even if $\mathbf{d}_{4}(z)$ is.

To solve this problem, let us consider the expression of $\mathbf{d}_{4}(z)$. By (12) and (35), we have

$$
\mathbf{d}_{4}(z)=\mathbf{e}_{4}(z) \mathbf{E}_{3}^{\dagger}(z)=\mathbf{e}_{4}(z) \mathbf{E}_{2}^{-1}(z)\left[\begin{array}{ccc}
\frac{1}{2} & 0 & \frac{1}{2} \\
0 & 1 & 0
\end{array}\right] .
$$

Then, define a $1 \times 2$ row vector $\mathbf{f}(z)$ as

$$
\mathbf{f}(z)=\mathbf{e}_{4}(z) \mathbf{E}_{2}^{-1}(z)\left[\begin{array}{cc}
\frac{\sqrt{2}}{2} & 0 \\
0 & 1
\end{array}\right]
$$



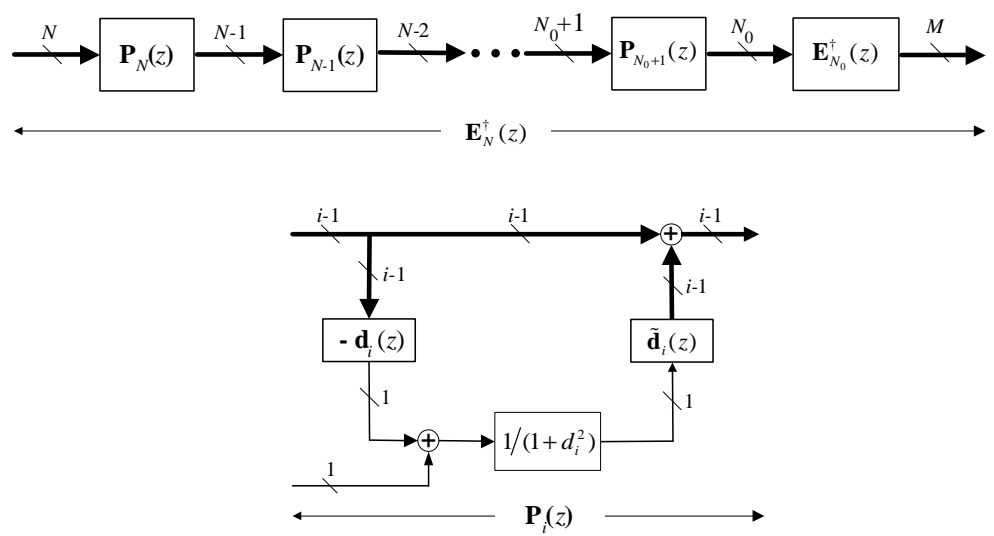

Fig. 2. Implementation diagram of $\mathbf{E}_{N}^{\dagger}(z)$ for a DFIR system.

and it can be verified that $\mathbf{d}_{4}(z) \tilde{\mathbf{d}}_{4}(z)=\mathbf{f}(z) \tilde{\mathbf{f}}(z)$, which indicates that $\mathbf{f}(z)$ is also a PU row vector. Taking the inverse transform of (36), we find

$$
\mathbf{e}_{4}(z)=\mathbf{f}(z)\left[\begin{array}{cc}
\sqrt{2} & 0 \\
0 & 1
\end{array}\right] \mathbf{E}_{2}(z) .
$$

Recall that for a PU row vector $\mathbf{f}(z)$ with degree of $K$, it can be completely characterized as [25]

$$
\mathbf{f}(z)=\mathbf{v}_{0} \mathbf{V}_{1}(z) \mathbf{V}_{2}(z) \cdots \mathbf{V}_{K}(z)
$$

where $\mathbf{v}_{0}$ is an arbitrary non-zero row vector and each $\mathbf{V}_{k}(z)$ $(1 \leq k \leq K)$ can be expressed as $\mathbf{V}_{k}(z)=\mathbf{I}-\mathbf{v}_{k}^{H} \mathbf{v}_{k}+$ $z^{-1} \mathbf{v}_{k}^{H} \mathbf{v}_{k}$, in which $\mathbf{v}_{k}$ is a row vector with unit norm, i.e., $\mathbf{v}_{k} \mathbf{v}_{k}^{H}=1$. Based on the above result, we know that $\mathbf{e}_{4}(z)$ can be designed by optimizing $\mathbf{v}_{k}$ for $k=1, \cdots, K$.

Not only does the Greville formula offer an effective way to design the DFIR system, it is also advantageous in terms of hardware implementation. $\mathbf{E}_{N}^{\dagger}(z)$ can be realized through a cascading structure as shown in Fig. 2, where the building blocks $\mathbf{P}_{i}(z)$ act as pre-filters of the input signal before it is passed to $\mathbf{E}_{N_{0}}^{\dagger}(z)$. Moreover, for a PU row vector $\mathbf{d}_{i}(z)$ satisfying (32), the VLSI-friendly implementation has been well established [25].

\section{Dual Frame of Laplacian Pyramids}

The derivation of the previous section is based on Case 1 of the forward Greville formula. In this section, Case 2 is applied to derive the dual frame of the Laplacian pyramid (LP) [22], which has been proved to be a useful tool for image processing and computer vision. Fig. 3 shows its implementation diagram, where $H(z)$ and $G(z)$ represent, respectively, the decimation and interpolation low-pass filters. The output signal is made up of two components: the coarse signal $x_{c}[n]$ represents the low-frequency components of the original input, while the details (with band-pass and high-pass frequency components) are contained in $x_{d}[n]$.

As $x[n]$ can be always reconstructed from $x_{c}[n]$ and $x_{d}[n]$, the LP realizes a frame expansion [23]. From the FB point of view, the LP can be implemented through an $(M+1)$-channel PR OFB with the sampling factor of $M$, whose polyphase matrix is given by [23]

$$
\mathbf{E}_{l p}(z)=\left[\begin{array}{c}
\mathbf{I}-\tilde{\mathbf{g}}(z) \mathbf{h}(z) \\
\mathbf{h}(z)
\end{array}\right],
$$

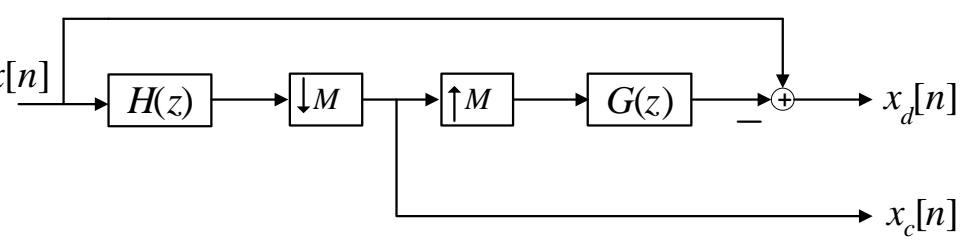

Fig. 3. Implementation diagram of Laplacian pyramid. Here, $H(z)$ and $G(z)$ represent the decimation and interpolation filters, respectively.

where the $1 \times M$ vectors $\mathbf{h}(z)$ and $\mathbf{g}(z)$ represent the TypeI polyphase matrices [25] of the low-pass filters $H(z)$ and $G(z)$, respectively. Although PR can be achieved for any pair of $H(z)$ and $G(z)$, a typical choice is to set $H(z)$ and $G(z)$ as biorthogonal pairs, in which $\mathbf{h}(z)$ and $\mathbf{g}(z)$ satisfy [23]

$$
\mathbf{h}(z) \tilde{\mathbf{g}}(z)=1 .
$$

Examples of biorthogonal filters include the $5 / 3$ and $9 / 7$ filters used in the JPEG 2000 standard and the original LP filters proposed in [22].

Our main purpose here is to derive a closed-form solution for $\mathbf{E}_{l p}^{\dagger}(z)$ under the biorthogonal condition (40). Denote $\mathbf{D}_{l p}(z)$ as the $M \times M$ submatrix of $\mathbf{E}_{l p}(z)$, i.e.,

$$
\mathbf{D}_{l p}(z)=\mathbf{I}-\tilde{\mathbf{g}}(z) \mathbf{h}(z) .
$$

When $\mathbf{h}(z)$ and $\tilde{\mathbf{g}}(z)$ satisfy (40), $\mathbf{D}_{l p}(z)$ is a rank-deficient matrix as one of its eigen-value is zero. Through some matrix manipulations, we can show

$\mathbf{D}_{l p}^{\dagger}(z)=\mathbf{I}_{M}-\frac{\tilde{\mathbf{g}}(z) \mathbf{g}(z)}{\mathbf{g}(z) \tilde{\mathbf{g}}(z)}-\frac{\tilde{\mathbf{h}}(z) \mathbf{h}(z)}{\mathbf{h}(z) \tilde{\mathbf{h}}(z)}+\frac{\tilde{\mathbf{g}}(z) \mathbf{h}(z)}{\mathbf{h}(z) \tilde{\mathbf{h}}(z) \mathbf{g}(z) \tilde{\mathbf{g}}(z)}$

It is not difficult to verify that $\mathbf{D}_{l p}^{\dagger}(z)$ satisfies the MoorePenrose equations. By exploiting the forward Greville formula, the LP's dual frame can be obtained as follows. Firstly, we have

$$
\mathbf{d}_{l p}(z)=\mathbf{h}(z) \mathbf{D}_{l p}^{\dagger}(z)=-\frac{\mathbf{g}(z)}{\mathbf{g}(z) \tilde{\mathbf{g}}(z)}+\frac{\mathbf{h}(z)}{\mathbf{h}(z) \tilde{\mathbf{h}}(z) \mathbf{g}(z) \tilde{\mathbf{g}}(z)}
$$

and

$$
\mathbf{c}_{l p}(z)=\mathbf{h}(z)-\mathbf{d}_{l p}(z) \mathbf{D}_{l p}(z)=\frac{\mathbf{g}(z)}{\mathbf{g}(z) \tilde{\mathbf{g}}(z)} .
$$

As $\mathbf{c}_{l p}(z)$ is not a constant zero vector (Case 2), we have

$$
\mathbf{r}_{l p}(z)=\frac{\mathbf{c}_{l p}(z)}{\mathbf{c}_{l p}(z) \tilde{\mathbf{c}}_{l p}(z)}=\mathbf{g}(z) .
$$


Then, $\mathbf{E}_{l p}^{\dagger}(z)$ can be computed from (16) as

$$
\mathbf{E}_{l p}^{\dagger}(z)=\left[\begin{array}{ll}
\mathbf{I}_{M}-\frac{\tilde{\mathbf{h}}(z) \mathbf{h}(z)}{\mathbf{h}(z) \tilde{\mathbf{h}}(z)} & \tilde{\mathbf{g}}(z)
\end{array}\right] .
$$

Up to now, we arrive at the following theorem:

Theorem 3: For $\mathbf{E}_{l p}(z)$ given in (39) with $\mathbf{h}(z) \tilde{\mathbf{g}}(z)=1$, its dual frame $\mathbf{E}_{l p}^{\dagger}(z)$ is given by (42).

Remark: When $H(z)$ is an orthonormal filter and $G(z)=$ $H(z)$, i.e., when $\mathbf{h}(z) \tilde{\mathbf{h}}(z)=1$ and $\mathbf{h}(z)=\mathbf{g}(z), \mathbf{E}_{l p}(z)$ is a PU matrix satisfying $\tilde{\mathbf{E}}_{l p}(z) \mathbf{E}_{l p}(z)=\mathbf{I}_{M}$. Under these restrictions, (42) boils down to $\mathbf{E}_{l p}^{\dagger}(z)=\left[\begin{array}{ll}\mathbf{I}_{M}-\tilde{\mathbf{h}}(z) \mathbf{h}(z) & \tilde{\mathbf{h}}(z)\end{array}\right]$, which is exactly $\tilde{\mathbf{E}}_{l p}(z)$ itself as expected.

Example 5 (Dual Frame of $L P$ ): To have another quick check of (42), consider an LP frame with $M=2$, where the decimation and the interpolation filters are scaled Haar filters with $H(z)=\frac{1}{2}\left(1+z^{-1}\right)$ and $G(z)=1+z^{-1}$, respectively. Here, we consider the scaled ones as they can be implemented using only shifts and adders without any multipliers. It is clear that $\mathbf{h}(z)=\frac{1}{2}\left[\begin{array}{ll}1 & 1\end{array}\right]$ and $\tilde{\mathbf{g}}(z)=\left[\begin{array}{ll}1 & 1\end{array}\right]^{T}$. Hence, by (39) and (42), $\mathbf{E}_{l p}(z)$ and $\mathbf{E}_{l p}^{\dagger}(z)$ are zero-order matrices, and are respectively given by

$$
\mathbf{E}_{l p}(z)=\frac{1}{2}\left[\begin{array}{ccc}
1 & -1 & 1 \\
-1 & 1 & 1
\end{array}\right]^{T}
$$

and

$$
\mathbf{E}_{l p}^{\dagger}(z)=\left[\begin{array}{ccc}
\frac{1}{2} & -\frac{1}{2} & 1 \\
-\frac{1}{2} & \frac{1}{2} & 1
\end{array}\right] .
$$

One can easily verify that $\mathbf{E}_{l p}^{\dagger}(z)$ is indeed the pseudo inverse of $\mathbf{E}_{l p}(z)$.

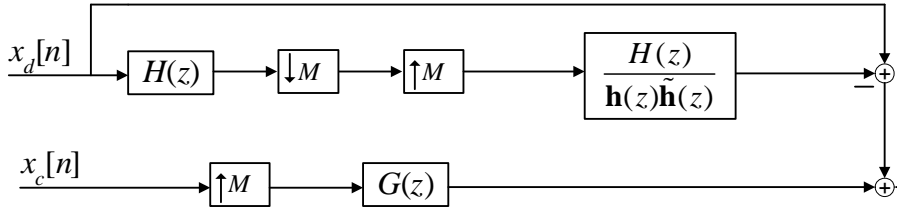

Fig. 4. Direct-form implementation diagram of the dual frame for the Laplacian pyramid.

The direct-form implementation of (42) is shown in Fig. 4. Note that in general, $\mathbf{E}_{l p}^{\dagger}(z)$ corresponds to an IIR filter bank. The necessary and sufficient condition for $\mathbf{E}_{l p}(z)$ to be DFIR is presented below:

Corollary 1: For an FIR $\mathbf{E}_{l p}(z)$ defined in (39) with $\mathbf{h}(z) \mathbf{g}(z)=1$, it is DFIR if and only if $\mathbf{h}(z)$ is a PU row vector satisfying $\mathbf{h}(z) \tilde{\mathbf{h}}(z)=\alpha$ for some positive constant $\alpha$.

Proof: The if part is obvious, to prove the "only if" part, let $\mathbf{S}_{l p}(z)=\mathbf{E}_{l p}^{\dagger}(z) \tilde{\mathbf{E}}_{l p}^{\dagger}(z)$. According to [18], $\mathbf{E}_{l p}(z)$ is FIR if and only if $\operatorname{det}\left(\mathbf{S}_{l p}(z)\right)=\alpha$ for some constant $\alpha$. It can be easily calculated that $\mathbf{S}_{l p}(z)=\mathbf{I}_{M}-\frac{\tilde{\mathbf{h}}(z) \mathbf{h}(z)}{\mathbf{h}(z) \tilde{\mathbf{h}}(z)}+\tilde{\mathbf{g}}(z) \mathbf{g}(z)$. Then, by matrix determinant lemma [26], $\operatorname{det}\left(\mathbf{S}_{l p}(z)\right)=$ $\frac{1}{\mathbf{h}(z) \tilde{\mathbf{h}}(z)}$, which completes the proof.

The above corollary implies that to get an FIR $\mathbf{E}_{l p}^{\dagger}(z)$, the decimation filter $H(z)$ should be an orthogonal one, while there is no restriction on the interpolation filter $G(z)$ except for the biorthogonal condition in (40).

In case when $\mathbf{E}_{l p}^{\dagger}(z)$ is IIR and we want to have an FIR approximation, we can replace the IIR filter $\frac{H(z)}{\mathbf{h}(z) \tilde{\mathbf{h}}(z)}$ in Fig. 4 with an FIR $K(z)$ satisfying

$$
K\left(e^{j \omega}\right) \approx \frac{H\left(e^{j \omega}\right)}{\mathbf{h}\left(e^{j \omega}\right) \tilde{\mathbf{h}}\left(e^{j \omega}\right)} .
$$

In terms of polyphase representation, it implies that $\mathbf{E}_{l p}^{\dagger}(z)$ is approximated by

$$
\mathbf{R}(z)=\left[\begin{array}{ll}
\mathbf{I}_{M}-\tilde{\mathbf{k}}(z) \mathbf{h}(z) & \tilde{\mathbf{g}}(z)
\end{array}\right],
$$

where $\mathbf{k}(z)$ is the $1 \times M$ polyphase vector of $K(z)$. With such an approximation, one can easily check that $\mathbf{R}(z) \mathbf{E}_{l p}(z)=$ $\mathbf{I}_{M}$, which indicates that the PR property is retained.

Example 6: Consider the 9/7 wavelet filter used in the JPEG 2000 standard. Since it is nearly orthogonal, we have $\mathbf{h}\left(e^{j \omega}\right) \tilde{\mathbf{h}}\left(e^{j \omega}\right) \approx 1$, which implies that we can set $K(z)=$ $H(z)$ with good approximation.

Remark: Curious readers may wonder how to calculate $\mathbf{E}_{l p}^{\dagger}(z)$ when $\mathbf{h}(z) \tilde{\mathbf{g}}(z) \neq 1$. In this scenario, $\mathbf{D}_{l p}(z)$ corresponds to a critically sampled PR FB, whose inverse is given by

$$
\mathbf{D}_{l p}^{-1}(z)=\mathbf{I}+\frac{1}{1-\mathbf{h}(z) \tilde{\mathbf{g}}(z)} \tilde{\mathbf{g}}(z) \mathbf{h}(z) .
$$

Then, $\mathbf{E}_{l p}(z)$ becomes an extended FB and accordingly, $\mathbf{E}_{l p}^{\dagger}(z)$ can be obtained from Case 1 of the forward Greville formula using (27).

\section{OFBS FOR ERASURE CHANNELS}

Due to the redundancy associated with frame expansions, OFBs can be used as joint source-channel codes to provide robustness to erasures [12]-[14]. In this section, we examine the resilience of OFBs in the presence of one erasure channel. The development is based on the backward Greville formula. To this end, we first introduce the following property [12]:

Let $\mathbf{E}_{N}(z)$ denote the $N \times M$ analysis polyphase matrix of PR OFB. Denote by $\mathbf{E}_{\{i\}}(z)$ the polyphase matrix obtained by deleting the $i$-th row of $\mathbf{E}_{N}(z) . \mathbf{E}_{N}(z)$ is robust to one erasure if $\mathbf{E}_{\{i\}}\left(e^{j \omega}\right)$ (for all $i=1, \cdots, N$ ) is of full rank on the unit circle.

\section{A. One Erasure}

This subsection studies the necessary and sufficient condition for OFBs to be robust to one erasure channel. Theorem 4 discusses the general PR OFBs, and Corollary 2 presents the special case of PU OFBs, or tight frames.

Theorem 4: Suppose that $\mathbf{E}_{N}(z)$ is the polyphase matrix of an $N$-channel PR OFB with its para-pseudo inverse given by $\mathbf{E}_{N}^{\dagger}(z)$. Let $\mathbf{e}_{i}(z)$ and $\tilde{\mathbf{r}}_{i}(z)$ (for $i=1, \cdots, N$ ) denote the $i$-th row vector of $\mathbf{E}_{N}(z)$ and the $i$-th column vector of $\mathbf{E}_{N}^{\dagger}(z)$, respectively. Then, $\mathbf{E}_{N}(z)$ is robust to one erasure if and only if

$$
\mathbf{e}_{i}\left(e^{j \omega}\right) \mathbf{r}_{i}^{H}\left(e^{j \omega}\right)<1
$$

for $i=1, \cdots, N$ and for all $\omega \in[0,2 \pi)$.

Proof: Note that when there is only one erasure channel, through row permutation, we can assume that the erasure occurs in the $N$-th channel. Let $\mathbf{E}_{N}(z)$ be written as in (11) with $i=N$. Without loss of generality, the proof is equivalent 
to showing that $\mathbf{E}_{N-1}\left(e^{j \omega}\right)$ in (11) has full rank if and only if

$$
\mathbf{e}_{N}\left(e^{j \omega}\right) \mathbf{r}_{N}^{H}\left(e^{j \omega}\right)<1 .
$$

For other values of $i$, we can just replace the subscript $N$ with $i$.

We start with the "only if" part. When $\mathbf{E}_{N-1}\left(e^{j \omega}\right)$ is of full rank, $\mathbf{e}_{N}\left(e^{j \omega}\right)$ lies in the row space of $\mathbf{E}_{N-1}\left(e^{j \omega}\right)$, which corresponds to Case 1 of the Greville formula. Let $\mathbf{d}_{N}\left(e^{j \omega}\right)=$ $\mathbf{e}_{N}\left(e^{j \omega}\right) \mathbf{E}_{N-1}^{\dagger}\left(e^{j \omega}\right)$. By (21), we have

$$
\begin{aligned}
\mathbf{e}_{N}\left(e^{j \omega}\right) \mathbf{r}_{N}^{H}\left(e^{j \omega}\right) & =\frac{\mathbf{d}_{N}\left(e^{j \omega}\right) \mathbf{d}_{N}^{H}\left(e^{j \omega}\right)}{1+\mathbf{d}_{N}\left(e^{j \omega}\right) \mathbf{d}_{N}^{H}\left(e^{j \omega}\right)} \\
& =\frac{\left\|\mathbf{d}_{N}\left(e^{j \omega}\right)\right\|^{2}}{1+\left\|\mathbf{d}_{N}\left(e^{j \omega}\right)\right\|^{2}}<1,
\end{aligned}
$$

which is exactly (45).

To prove the "if" part, notice that $\mathbf{e}_{N}\left(e^{j \omega}\right)$ must lie in the row space of $\mathbf{E}_{N-1}\left(e^{j \omega}\right)$ for all $\omega$, because otherwise, according to the backward Greville formula, there must exist some $\omega_{0}$ such that $\mathbf{e}_{N}\left(e^{j \omega_{0}}\right) \mathbf{r}_{N}^{H}\left(e^{j \omega_{0}}\right)=1$. Also, using the PR property of $\mathbf{E}_{N}(z)$, we have

$$
\operatorname{rank}\left(\mathbf{E}_{N-1}\left(e^{j \omega}\right)\right)=\operatorname{rank}\left(\mathbf{E}_{N}\left(e^{j \omega}\right)\right)=M,
$$

which indicates that $\mathbf{E}_{N-1}\left(e^{j \omega}\right)$ is of full rank on the unit circle.

Remark: As pointed out by one of the reviewers, the above result is consistent with the classical regression analysis. Note that $\mathbf{E}_{N}\left(e^{j \omega}\right) \mathbf{E}_{N}^{\dagger}\left(e^{j \omega}\right)$ is a projection matrix on the column space of $\mathbf{E}_{N}\left(e^{j \omega}\right)$, whose $i$-th diagonal element $\mathbf{e}_{i}\left(e^{j \omega}\right) \mathbf{r}_{i}^{H}\left(e^{j \omega}\right)$ is bounded by $0 \leq \mathbf{e}_{i}\left(e^{j \omega}\right) \mathbf{r}_{i}^{H}\left(e^{j \omega}\right) \leq 1$. Recall that $\mathbf{e}_{i}\left(e^{j \omega}\right) \mathbf{r}_{i}^{H}\left(e^{j \omega}\right)$ measures the importance of a given data dimension in regression analysis, the so called "leverage". Theorem 4 implies that if $\mathbf{E}_{N}(z)$ is not robust to one erasure, then there exists at least one $i$ satisfying $\mathbf{e}_{i}\left(e^{j \omega}\right) \mathbf{r}_{i}^{H}\left(e^{j \omega}\right)=1$, indicating that this dimension has too high a leverage - if it is lost, the sub-band signals cannot be projected back onto the original subspace through para-pseudo inverse.

Example 7: Consider the LP frame depicted in Fig. 3 with biorthogonal filters $H(z)$ and $G(z)$. From (39) and (42), we can easily verify that

$$
\mathbf{E}_{l p}(z) \mathbf{E}_{l p}^{\dagger}(z)=\left[\begin{array}{cc}
\mathbf{I}_{M}-\frac{\tilde{\mathbf{h}}(z) \mathbf{h}(z)}{\mathbf{h}(z) \hat{\mathbf{h}}(z)} & 0 \\
0 & 1
\end{array}\right] .
$$

This indicates that $\mathbf{e}_{N}(z) \tilde{\mathbf{r}}_{N}(z)=1$. By Theorem 4, the LP frame is not robust to one erasure channel when using biorthogonal filters.

For the special case when $\mathbf{E}_{N}(z)$ implements a tight frame with frame bound $A>0$, i.e., when $\mathbf{E}_{N}(z)$ is a PU matrix satisfying $\tilde{\mathbf{E}}_{N}(z) \mathbf{E}_{N}(z)=A \mathbf{I}_{M}$, its dual frame can be simply written into $\mathbf{E}_{N}^{\dagger}(z)=\frac{1}{A} \tilde{\mathbf{E}}(z)$. A consequence of Theorem 4 is as follows:

Corollary 2: If $\mathbf{E}_{N}(z)$ corresponds to a tight frame with frame bound $A$, it is robust to one erasure channel if and only if its $i$-th row vector $\mathbf{e}_{i}(z)$ satisfies

$$
\mathbf{e}_{i}\left(e^{j \omega}\right) \mathbf{e}_{i}^{H}\left(e^{j \omega}\right)<A
$$

for all $i=1, \cdots, N$ and for all $\omega \in[0,2 \pi)$.

Remarks:

1) Note that [12] also investigated the scenario of one erasure. But the discussions there were focused on the uniform tight frame (UTF), a special class of tight frames with equal norm for each analysis filter. Our derivations are for general frames implemented via PR and PU OFBs. It can be shown that when $A=N / M$, Corollary 2 boils down to Theorem 5 in [12]. Although theoretically, UTFs provide optimal performance, their design is rather difficult. On the other hand, several works have reported simple design methods and fast implementations for cosine modulated OFBs [7] and linear-phase OFBs [10], which are attractive in practical applications like orthogonal frequency-division multiplexing (OFDM) and image coding. The theory developed here can be used for those FBs which do not generate UTFs.

2) Upon completion of this journal paper, we became aware of the parallel work [28] that also proved Corollary 2 (a special case of Theorem 4) using a different approach.

\section{B. Implementation Structure}

In this subsection, we consider the following problem: when the erasure occurs in one channel, how to efficiently compute and implement the para-pseudo inverse of the remaining analysis bank? Note that a naive approach is to directly compute the para-pseudo inverse, which would cause unnecessarily high complexity. The use of the backward Greville formula gives a low-complexity solution.

Again, without loss of generality, let us assume that the subband coefficients in the $N$-th channel are completely lost. From (23)-(25), it is straightforward to see

$$
\mathbf{E}_{N-1}^{\dagger}(z)=\left(\mathbf{I}_{M}+\mathbf{Q}(z)\right) \mathbf{E}_{N}^{\dagger}(z)\left[\begin{array}{c}
\mathbf{I}_{N-1} \\
\mathbf{0}
\end{array}\right],
$$

where the $M \times M$ matrix $\mathbf{Q}(z)$ takes the form of

$$
\mathbf{Q}(z)= \begin{cases}\frac{\tilde{\mathbf{r}}_{N}(z) \mathbf{e}_{N}(z)}{1-\mathbf{e}_{N}(z) \tilde{\mathbf{r}}_{N}(z)}, & \text { Case 1; } \\ -\frac{\tilde{\mathbf{r}}_{N}(z) \mathbf{r}_{N}(z)}{\mathbf{r}_{N}(z) \tilde{\mathbf{r}}_{N}(z)}, & \text { Case 2. }\end{cases}
$$

Note that in Case $1, \mathbf{E}_{N-1}(z)$ generates a frame expansion and thus, $\mathbf{E}_{N-1}^{\dagger}(z)$ corresponds to its dual frame. While it does not in Case 2, $\mathbf{E}_{N-1}^{\dagger}(z)$ still nonetheless leads to a minimum $l_{2}$ norm reconstruction. It can be readily seen that the para-pseudo inverse of the remaining FB is in general a IIR system. The design of oversampled FB yielding stable and causal para-pseudo inverse for erasure channels remains as an open question.

Fig. 5 shows the corresponding implementation structure. The process can be described as follows. First, in the frequency domain, the subband coefficients in the erasure channel (i.e., the $N$-th channel) are set to zeros. Then, the original dual frame $\mathbf{E}_{N}^{\dagger}(z)$ is applied, followed by a time-domain post-filter $\mathbf{I}_{M}+\mathbf{Q}(z)$ to yield the reconstructed signal. In essence, $\mathbf{Q}(z)$ is used to compensate for the erasure in the $N$-th channel. One can see that the implementation in Fig. 5 has the time-domain 


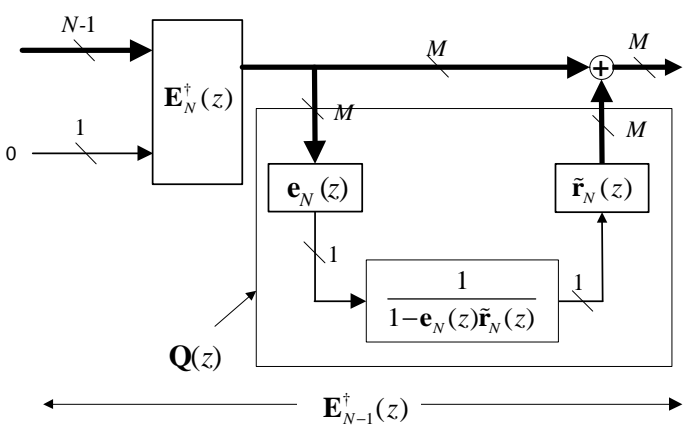

(a)

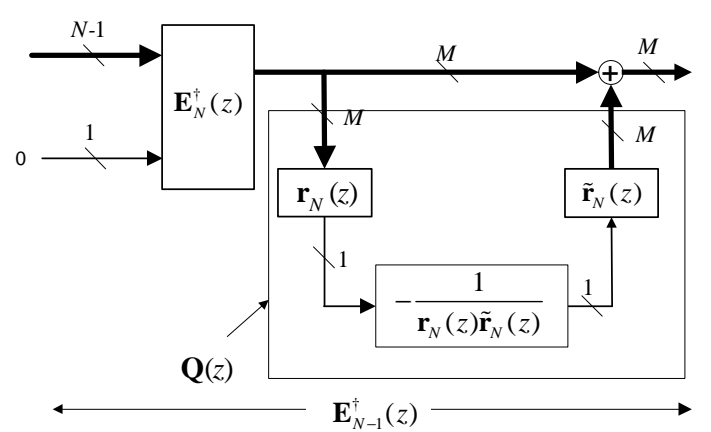

(b)

Fig. 5. Post-filtering implementation structure for $\mathbf{E}_{N-1}^{\dagger}$ (z). (a) Case 1; (b) Case 2.

post-processing structure. For Case 1, an alternative way to implement $\mathbf{E}_{N-1}^{\dagger}(z)$ was proposed in [13], where the lost subband coefficients are first predicted in the frequency domain before being reconstructed by $\mathbf{E}_{N}^{\dagger}(z)$. It can be shown that these two structures have about the same the implementation complexity. One attractive property of our proposed structure is that it can be applied to time-domain over-sampled lapped transforms (TDOLTs) [29], which admits a similar structure of pre-/post-filtering outside the DCT and the IDCT. In this way, existing DCT-based standards (like the JPEG) are kept intact. One of our on-going works is to integrate (47) with TDOLTs for joint source-channel coding.

\section{CONCLUSIONS}

We have studied the computation of the para-pseudo inverse via the Greville formulas. The forward Greville formula was utilized to compute the para-pseudo inverse of a matrix when an additional row is appended. We then derived the backward Greville formula to deal with row deletion. The applications of forward and backward Greville formulas were demonstrated in frame and OFB theories. In particular, we applied the forward Greville formula (Case 1) to study an extended FB, where we presented an iterative way to calculate its dual frame. The necessary and sufficient condition to generate DFIR systems from extended FBs was also derived. Besides, the Greville formula (Case 2) led to a closed-form solution of the dual frame for Laplacian pyramids. Based on the backward Greville formula (Case 1), we derived the necessary and sufficient condition for PR OFBs to be robust to one erasure channel. We also proposed a post-filtering structure to implement the dual frame in the presence one erasure channel. The scenario of more than one erasures will be investigated in the future.

It should also be noted that Case 2 of the backward Greville formula has been used in V-BLAST detection, where the channel matrix is of full rank [30]. Finally, it follows from Naimark's theorem [15], [16] that the $N \times M(N>M)$ polyphase matrix of a PR OFB can be obtained by deleting $N-M$ columns of an $N \times N$ critically-sampled one. The same procedure as presented in [30] can easily be applied to obtain the dual frame of such an OFB.

\section{APPENDIX \\ ProOF OF BACKWARD GREVILLE FormULA}

Let $\mathbf{E}_{i}(z)$ and $\mathbf{E}_{i}^{\dagger}(z)$ be partitioned as in (11) and (20), respectively. From (16), we can easily get the expression of $\mathbf{E}_{i-1}^{\dagger}(z)$ as in (25), which means $\mathbf{E}_{i-1}^{\dagger}(z)$ can be computed if $\mathbf{d}_{i}(z)$ can be expressed in terms of $\mathbf{E}_{i}^{\dagger}(z)$ and $\mathbf{E}_{i}(z)$, or parts of them.

In Case 1, we multiply $\mathbf{R}_{i-1}(z)$ with $\mathbf{e}_{i}(z)$, yielding

$$
\begin{aligned}
\mathbf{e}_{i}(z) \mathbf{R}_{i-1}(z) & =\mathbf{e}_{i}(z) \mathbf{E}_{i-1}^{\dagger}(z)-\mathbf{e}_{i}(z) \tilde{\mathbf{r}}_{i}(z) \mathbf{d}_{i}(z) \\
& =\mathbf{d}_{i}(z)-\mathbf{e}_{i}(z) \tilde{\mathbf{r}}_{i}(z) \mathbf{d}_{i}(z) \\
& =\left(1-\mathbf{e}_{i}(z) \tilde{\mathbf{r}}_{i}(z)\right) \mathbf{d}_{i}(z) .
\end{aligned}
$$

Then,

$$
\mathbf{d}_{i}(z)=\frac{\mathbf{e}_{i}(z) \mathbf{R}_{i-1}(z)}{1-\mathbf{e}_{i}(z) \tilde{\mathbf{r}}_{i}(z)}
$$

which, as expected, is expressed in known $\mathbf{R}_{i-1}(z), \mathbf{e}_{i}(z)$, and $\tilde{\mathbf{r}}_{i}(z)$.

In Case 2, we multiply $\mathbf{R}_{i-1}(z)$ with $\mathbf{r}_{i}(z)$, yielding

$$
\mathbf{r}_{i}(z) \mathbf{R}_{i-1}(z)=\mathbf{r}_{i}(z) \mathbf{E}_{i-1}^{\dagger}(z)-\mathbf{r}_{i}(z) \tilde{\mathbf{r}}_{i}(z) \mathbf{d}_{i}(z)
$$

Note that as $\mathbf{r}_{i}(z)=\frac{\mathbf{c}_{i}(z)}{\mathbf{c}_{i}(z) \tilde{\mathbf{c}}_{i}(z)}$, we have

$$
\mathbf{r}_{i}(z) \mathbf{E}_{i-1}^{\dagger}(z)=\frac{1}{\mathbf{c}_{i}(z) \tilde{\mathbf{c}}_{i}(z)} \mathbf{c}_{i}(z) \mathbf{E}_{i-1}^{\dagger}(z)
$$

Based on (12), (13) and the Moore-Penrose condition (2), we can get

$$
\begin{aligned}
\mathbf{c}_{i}(z) \mathbf{E}_{i-1}^{\dagger}(z) & =\mathbf{e}_{i}(z) \mathbf{E}_{i-1}^{\dagger}(z)-\mathbf{d}_{i}(z) \mathbf{E}_{i-1}(z) \mathbf{E}_{i-1}^{\dagger}(z) \\
& =\mathbf{e}_{i}(z) \mathbf{E}_{i-1}^{\dagger}(z)-\mathbf{e}_{i}(z) \mathbf{E}_{i-1}^{\dagger}(z) \mathbf{E}_{i-1}(z) \mathbf{E}_{i-1}^{\dagger}(z) \\
& =0
\end{aligned}
$$

which indicates that $\mathbf{r}_{i}(z) \mathbf{E}_{i-1}^{\dagger}(z)=0$. Substituting it into (50) yields

$$
\mathbf{r}_{i}(z) \mathbf{R}_{i-1}(z)=-\mathbf{r}_{i}(z) \tilde{\mathbf{r}}_{i}(z) \mathbf{d}_{i}(z)
$$

which produces the desired expression

$$
\mathbf{d}_{i}(z)=-\frac{\mathbf{r}_{i}(z) \mathbf{R}_{i-1}(z)}{\mathbf{r}_{i}(z) \tilde{\mathbf{r}}_{i}(z)} .
$$




\section{ACKNOWLEDGMENT}

The authors would like to thank Dr. Peter J. Schreier from University of Newcastle, Australia for generously sharing the course notes [26]. We also want to thank the anonymous reviewers for their thorough reviews and numerous constructive suggestions which significantly enhance the presentation of the paper.

\section{REFERENCES}

[1] R. J. Duffin and A. C. Schaeffer, "A class of nonharmonic Fourier series," Trans. Amer. Math. Soc., vol. 72, pp. 341-366, 1952.

[2] I. Daubechies, A. Grossman, and Y. Meyer, "Painless nonorthogonal expansions," J. Math. Phys., vol. 27, pp. 1271-1283, Nov. 1986.

[3] Z. Cvetković and M. Vetterli, "Oversampled filter banks," IEEE Trans. Signal Processing, vol. 46, pp. 1245-1255, May 1998.

[4] H. Bölcskei, F. Hlawatsch, and H. G. Feichtinger, "Frame-theoretic analysis of oversampled filter banks," IEEE Trans. Signal Processing, vol. 46, pp. 3256-3268, Dec. 1998.

[5] K. F. C. Yiu, N. Grbic, S. Nordholm, and K. L. Teo, "Multicriteria design of oversampled uniform DFT filter banks," IEEE Signal Processing Lett., vol. 11, pp. 541-544, June 2004.

[6] M. F. Mansour, "On the optimization of oversampled DFT filter banks," IEEE Signal Processing Lett., vol. 14, pp. 389 - 392, June 2007.

[7] H. Bölcskei and F. Hlawatsch, "Oversampled cosine modulated filter banks with perfect reconstruction," IEEE Trans. Circuits Syst. II, vol. 45, pp. 1057-1071, Aug. 1998.

[8] J. Kliewer and A. Mertins, "Oversampled cosine-modulated filter banks with arbitrary system delay," IEEE Trans. Signal Processing, vol. 46, pp. 941-955, Apr. 1998.

[9] F. Labeau and L. Vandendorpe, "Structures, factorizations, and design criteria for oversampled paraunitary filterbanks yielding linear-phase filters," IEEE Trans. Signal Processing, vol. 48, pp. 3062-3071, Nov. 2000.

[10] L. Gan and K.-K. Ma, "Oversampled linear-phase perfect reconstruction filterbanks: Theory, lattice structure and parameterization," IEEE Trans. Signal Processing, vol. 51, pp. 744-759, Mar. 2003.

[11] T. Tanaka and Y. Yamashita, "The generalized lapped pseudobiorthogonal transform: Oversampled linear-phase perfect reconstruction filter banks with lattice structures," IEEE Trans. Signal Processing, vol. 52, pp. 434-446, Feb. 2004.

[12] J. Kovačević, P. L. Dragotti, and V. K. Goyal, "Filter bank frame expansions with erasures," IEEE Trans. Inform. Theory, vol. 48, pp. 1439-1450, June 2000.

[13] R. Bernardini and R. Rinaldo, "Efficient reconstruction from framebased multiple descriptions," IEEE Trans. Signal Processing, vol. 53, pp. 3282-3296, Aug. 2005

[14] T. Tanaka, "A direct design of oversampled perfect reconstruction FIR filter banks of 50\%-overlapping filters," IEEE Trans. Signal Processing, vol. 54, pp. 3011-3022, Aug. 2006.

[15] J. Kovačević and A. Chebira, "Life beyond bases: The advent of frames (part i)," IEEE Signal Processing Mag., vol. 24, pp. 86-104, 2007.

[16] _ "Life beyond bases: The advent of frames (part ii)," IEEE Signal Processing Mag., vol. 24, pp. 115-125, 2007.

[17] L. Chai, J. Zhang, and C. Zhang, "Frame-theory based analysis and design of oversampled filter banks: Direct computational method," IEEE Trans. Signal Processing, vol. 55, pp. 507-519, Feb. 2007.

[18] R. Bernardini and R. Rinaldo, "Oversampled filter banks from extended perfect reconstruction filter banks," IEEE Trans. Signal Processing, vol. 54, pp. 2625-2635, 2006.

[19] T. N. E. Greville, "Some applications of the pseudo inverse of a matrix," SIAM Review, vol. 2, pp. 15-22, 1960.

[20] S. Mohideen and V. Cherkassky, "On recursive calculation of the generalized inverse of a matrix," ACM Transactions on Mathematical Software, vol. 17, pp. 130-147, Mar. 1991.

[21] P. L. Dragotti, S. Servetto, and M. Vetterli, "Filter banks for multiple description coding: Analysis and synthesis," IEEE Trans. Inform. Theory, vol. 48, pp. 2036-2052, July 2000.

[22] P. J. Burt and E. H. Adelson, "The Laplacian pyramid as a compact image code," IEEE Trans. Commun., vol. 31, pp. 532-540, Apr. 1983.

[23] M. N. Do and M. Vetterli, "Framing pyramids," IEEE Trans. Signal Processing, vol. 51, pp. 2329-2342, Sept. 2003.

[24] R. A. Horn and C. R. Johnson, Matrix Analysis. New York, NY: Cambridge Univ. Press, 1991.
[25] P. P. Vaidyanathan, Multirate Systems and Filter Banks. Englewood Cliffs, NJ: Prentice-Hall, 1993.

[26] C. T. Mullis, Advanced Linear Systems. University of Colorado, 2000, course Notes.

[27] R. Fletcher, "A technique for orthogonalization," J. Inst. Math. Appl., vol. 5, pp. 162-166, 1969

[28] Z. Xu and A. Makur, "On the robustness of filter bank frame to quantization and erasures," in Proc. IEEE ICASSP 2007, Honolulu, Hawaii, USA, Apr. 2007.

[29] L. Gan and K.-K. Ma, "Time-domain oversampled lapped transforms: Theory, structure and application in image coding," IEEE Trans. Signal Processing, vol. 52, pp. 2762-2775, Oct. 2004.

[30] C. Ling, L. Gan, and W. H. Mow, "A dual-lattice view of V-BLAST detection," in IEEE Information Theory Workshop (ITW), Chengdu, China, Oct. 2006. 